\title{
Why sharing data and code during peer review can enhance behavioral ecology research
}

\author{
Esteban Fernández-Juricic ${ }^{1}$
}

Published online: 25 June 2021

(c) The Author(s), under exclusive licence to Springer-Verlag GmbH Germany, part of Springer Nature 2021

We live in the times of a reproducibility crisis in science (Open Science Collaboration 2015; Baker 2016; National Academies of Sciences, Engineering, and Medicine 2019). Even Ecology and Evolutionary Biology have been shown to be affected by many of the factors involved in decreasing research transparency and enhancing publication bias (Fidler et al. 2017; Fraser et al. 2018; O'Grady 2020). Regrettably, we do not have enough replication studies in Ecology and Evolutionary Biology to quantify the extent of the reproducibility problem (Kelly 2019; Fraser et al. 2020), but those published to date underscore potential issues (Clark et al. 2020a, b; Munday et al. 2020). It appears that Behavioral Ecology is not impervious to the reproducibility crisis (Parker 2013; Ilhe et al. 2017; Stevens 2017; Farrar et al. 2020).

There have been many calls and specific suggestions to revert reproducibility issues (Hampton et al. 2015; Munafo et al. 2017). One of the strategies to improve research transparency is computational and statistical reproducibility (Buckheit and Donoho1995; National Academies of Sciences, Engineering, and Medicine 2019), which has been specifically considered in many ecological journals (Mislan et al. 2016; Archmiller et al. 2020; Culina et al. 2020). The idea is to make available not just the data but also the code (in R, Python, SAS, etc.) used to process raw data, simulate data, run statistical analyses, and develop figures so that readers can generate the same outcomes as those presented in the paper (Sandve et al. 2013; Ilhe et al. 2017; Perkel 2019; Powers and Hampton 2019). Many ecological journals are already asking authors to make their data available with the publication of a paper, and authors appear to have embraced this practice (about $79 \%$ of papers comply; Culina et al. 2020), although a large proportion of these datasets

Esteban Fernández-Juricic

efernan@purdue.edu

1 Department of Biological Sciences, Purdue University, 915 W, State Street, West Lafayette, IN 47907, USA appear incomplete to be reused (Roche et al. 2015). Some other journals have also made mandatory or encouraged the availability of code with the publication, but authors appear to not have followed this practice as much (only about $27 \%$ of papers comply; Culina et al. 2020). Other papers report similar trends in Ecology (Mislan et al. 2016; Archmiller et al. 2020).

Powers and Hampton (2019) argue that data and code should actually become available during (instead of after) the peer review process. The motivation is to provide reviewers the opportunity to inspect the data structure, the code, and even run it if they wish to do so. There are two components about reviewing the code: first, to ensure that the code provided by the authors produces the same results as those reported in the manuscript; second, to ensure that the statistical analyses used in the code are aligned with the experimental design and structure of the data provided by the authors (i.e., model assumptions tested, appropriate data transformations, formulation of the statistical model matching the temporal and spatial structure of the data points, correct interpretation of statistical model coefficients, P-values, and effect sizes). The benefits of reviewing data/code are not minor (Ilhe et al. 2017; Powers and Hampton 2019): (a) reviewers are provided with a new dimension in the evaluation of the strengths and weaknesses of the experimental design, data collected, and analyses conducted; (b) reviewers can then provide very specific comments to help authors improve the quality of the manuscript; (c) editors can optimize the limited availability of reviewers' time by better establishing the fit of a manuscript to a given journal from a statistical viewpoint; and (d) the overall research transparency of a discipline can be enhanced. Multiple guidelines have been proposed to implement computational and statistical reproducibility (Noble 2009; Hampton et al. 2015; Mislan et al. 2016; Wilkinson et al. 2016; Cooper and Hsing 2017; Ilhe et al. 2017; Powers and Hampton 2019; Van Lissa et al. 2020). 
There is something very important for the whole community to realize: allowing data and code to be available during peer review is not intended to identify scientific fraud. It is actually intended to enhance the quality of a manuscript! More specifically, the motivation is to ensure that the experimental design, structure of the data, and statistical analyses are all aligned logically. Reviewers may have no problem assessing that alignment with simple experimental designs, simple data sets, and simple statistical analysis (e.g., independent sample t-tests). Yet, the same task may be much more challenging with more complex experimental designs (e.g., repeated measures) and statistical analysis (e.g., mixed models) often used in Behavioral Ecology. When manuscripts provide little detail about their statistical analyses (e.g., "We analyzed our data with a linear mixed model."), reviewers are placed in a very difficult position that sometimes leads to an unproductive cycle: reviewers may not respond favorably to the manuscript, which deeply frustrates authors.

Yet sharing both data and code for peer review is not easy. In my lab, we have spent almost 2 years figuring out strategies to implement computational and statistical reproducibility in our workflows. We transitioned to a point in which besides writing a manuscript, we spend days/weeks writing code that can be understood and run by others. Adopting this approach obviously adds to the already long to-do lists of scientists who are already overworked and stressed with deadlines and the high level of competition to publish and get funded. The counterpoint is that the process and the outcome can be quite rewarding. We have learned a lot about bottlenecks in the lab's workflows that made computational and statistical reproducibility challenging. We are now trying to bridge the gap in data curation and statistical procedures between former and current lab members, and it will probably take a few years to close this gap. However, moving forward, we strive to have data plus code ready for the submission of any manuscript irrespective of whether the journal makes it mandatory or not.

After all this hard work, I started noticing that my reference points for reviewing manuscripts (our own and others) shifted in a dramatic fashion. Now I have a difficult time critically reviewing a manuscript without having extremely easy access to the data and the code used. For instance, by looking at the code, I can easily establish whether (and most importantly how) the assumptions of statistical models were checked and what was done to deal with assumption violations without having to ask authors for more details every time I get a new version or having to assume that it was done this or that way. Having access to code plus data has sharpened my ability to find the strengths and weaknesses of a manuscript and offer a very detailed roadmap on how authors can make the best out of the data they gathered.
Given this paradigm shift, I have started requesting data plus code from both authors and collaborators in the last few months. Authors argued that they might be willing to make data and code available after the official acceptance of the manuscript. This answer appears rooted in the perception that their data and/or code could be "scooped." Although understandable, this view is not aligned with the reality of mandatory open data (in some cases enforced by both funding agencies and journals) and open platforms (R, Python, etc.) scientists are embracing worldwide (Lai et al. 2019; http://r4stats.com/articles/popularity/). When journals request sharing data, authors can use a repository or an electronic supplementary file during submission. When it comes to code, using open code from others (provided the source is acknowledged) is accepted in some scientific communities to maintain analytical standards or even improve them (Barnes 2010; Perkel 2019). Sharing data and/or code in certain repositories will generate a DOI (Mislan et al. 2016; https://guides.github.com/activities/citable-code/), ensuring authors have an official record of their work, and ultimately decreasing the chances of scooping. More importantly, the peer review process is the moment to assess that computational and statistical robustness of the study to find areas for improvement. For instance, the three times I have asked authors to share data and code as a reviewer/editor, I found that the code did not match the structure of the data and when fixed, the results were slightly to very different from the ones originally reported in the first version of the manuscript. Although no conclusion can be drawn from a sample size of 3, the contention still stands: reviewer-suggested modifications of the statistical analyses could change the results and their discussion in major ways. If those modifications in analyses/interpretation are major and occur after publication, it could even lead to the retraction of papers (examples: https://retractionwatch.com/), questioning the credibility of not only authors but also reviewers/editors (Anonymus 2020; Wessel 2020).

When I asked collaborators about sharing data and code, their responses showed an extremely high degree of variation: from receiving them right away to different levels of reticence, including initial second guessing, and even declining the request altogether arguing it was offending. The latter response is also understandable. Authors pour their hearts and souls into developing their experimental designs, deciding on specific statistical tests, processing data, running the analyses, etc. When a collaborator out of the blue asks to see the data and code, it can lead to perceptions of mistrust. Yet, this can also be an opportunity to educate ourselves and colleagues. First, when setting up a research collaboration, we ought to lay out the expectations on computational and statistical reproducibility between labs. My lab now states that whatever data and code we generate, we will share them (whether requested or not) as new versions are developed 
and that we expect the other lab/s to re-run it to make sure we are on the same analytical page. We also mention that we would appreciate this to be reciprocated during the collaboration. For collaborations already established, our strategy is now to explain the value of computational and statistical reproducibility, attaching the following papers (Mislan et al. 2016; Cooper and Hsing 2017; Powers and Hampton 2019), and eventually kindly asking for data and code. These strategies have smoothed out many of the negative responses we used to get. More importantly, running the code of collaborators has led to strengthening the connections with them. For instance, we now have virtual sessions running sections of code that are unclear, which often turn into excellent conversations about model assumptions, data transformation, statistical model set-up, and even interpretations of the results. These sessions have become great learning resources for everyone involved. When grad students and post-docs participate, these sessions help them better understand the mechanics and value of research collaborations between labs.

Editors also have concerns about encouraging (or making it mandatory for) authors to share data and code at the moment of submission. The argument is that it is already difficult to find reviewers to read manuscripts; thus, burdening reviewers even more to also assess code and data could unbalance the already strained peer-review process (Hatchwell 2017). This is a fair comment, but it needs to be considered in a broader context. One of the main reasons behind promoting computational/statistical reproducibility during peer review is to strengthen the analytical support of the results of a study. If a journal does not make reviewing data/code even an option, we run the risk that manuscripts published in that journal might not have reached their peak in computational and statistical quality. This could hurt the reputation of a journal. Ultimately, this dilemma can be thought of as a trade-off between reviewers' time allocation and quality of published papers. There are several non-mutually exclusive strategies to embrace a culture of reviewing data/code by reducing the high implementation costs per reviewer: (a) adopting volunteer/paid code editors for only complex statistical procedures (e.g., mixed models, neural networks, etc.), (b) encouraging (rather than expecting) reviewers to also take a look at the code if willing to do so, (c) adding a reviewer to only go over the code on top of the two often asked to review the whole manuscript, (d) encouraging authors to upload pre-prints before submission with data and code to get comments from the community and submitting those comments related to data/code implementation (and the authors' answers) as part of the submission process, (e) requiring authors to get a certificate on code reproducibility before submission via https://codecheck. org.uk/ to ensure their code can be run successfully by anybody, (f) encouraging authors to have a colleague (not associated with the authorship of the manuscript) run the code for reproducibility purposes and provide their name and contact info as part of the submission process, etc. The main idea is that the more people see the authors' code, the higher the chances of improving it (Ilhe et al. 2017). That in itself can enhance the overall quality of manuscripts published in a given journal, benefiting the whole discipline.

For the whole community to buy in the submission of data and code for peer review, editorial offices should consider changing the way manuscripts are evaluated. One of the fears authors may have is that reviewers' suggestions related to data processing and data analysis may change the results of the study; and consequently, the interest of the journal in further considering the manuscript for publication. This fear stems from a culture in which the chances of acceptance rely heavily on "beautiful results" rather than on the strength of the experimental design addressing a given question. As many advocates of open science have stated, we need to focus on "beautiful methods" rather than "beautiful results" (Nosek et al. 2012). This might not be a popular editorial shift in some journals, but it has the potential to strengthen our discipline by providing authors some certainty that modification of the results (as long as they have a well-defined question followed by a strong experimental design) through the peer review of their code will not be penalized. This is not a minor detail as publishing is still an important component of advancing a scientist's career.

There are a few additional arguments that can be made to hopefully tip the balance in the direction of submitting data and code for peer review in the context of research reproducibility.

First, once a lab embraces a culture of sharing data and code for peer review, its publication rate may increase because unpublished manuscripts by former students may be easier to submit by PIs and new grad students given the clear understanding of how the data were collected and analyzed in the past. This is not usually the case when labs do not have high standards of computational and statistical reproducibility, leading to datasets that are never published, which hurts both the lab as well as the entire discipline.

Second, making the data and code accessible for peer review may facilitate the work of reviewers in major ways, which might lead to more positive decisions about revisions (instead of plain rejections), boosting the chances of eventual acceptance of a manuscript. Obviously, this line of logic would require proper empirical testing.

Third, sharing data and code for peer review provides an opportunity for the analytical foundations of a study to stand out on themselves rather than the name and/or prestige of some principal investigators and/or institutions. This can 
promote diversity and inclusion goals in academic environments (Grahe et al. 2019).

Fourth, preparing the data and code for peer review can facilitate the process of making it open right after the manuscript is accepted. This in turn can lead into the data and code to be more easily reused by the community, increasing the chances of new collaborations, boosting paper citation rates (Piwowar et al. 2007; Piwowar and Vision 2013; McKiernan et al. 2016; Christensen et al. 2019; Colavizza et al. 2020), and making it possible for others to add the results of the study in meta-analyses as effect sizes and measures of variation can be more easily extracted. Additionally, papers with easily accessible data and code can get greater exposure to undergraduate and graduate students taking statistics courses as instructors are often in search of papers with open data and code to provide real-life examples in their classes. Ultimately, open data and code can be thought of as a learning resource for early career researchers in our discipline.

The take-home message is that making data and code available for peer review (and reviewing it) does have implementation costs; however, the multiple benefits may outweigh these costs. Editors of journals who publish behavioral ecology papers have the opportunity to lead the way towards shifting research practices and make transformative and lasting changes. There are some journals that are taking the necessary steps for data/code availability during peer reviews, such as Behavioral Ecology and Sociobiology (Bakker and Traniello 2020). The hope is that we soon move to a new standard in peer review in the behavioral sciences. It is a timely and healthy move for Behavioral Ecology.

Acknowledgements I truly thank Theo Bakker, James Traniello, Susan Healy, Nancy Solomon, Tim Parker, and Brain Nosek for sharing their views and input on this topic and my lab members for embracing the transition into computational and statistical reproducibility.

\section{References}

Anonymus (2020) Editorial: regarding mentorship. Nat Commun 11:6447. https://doi.org/10.1038/s41467-020-20618-x

Archmiller AA, Johnson AD, Nolan J, Edwards M, Elliott LH, Ferguson JM, Iannarilli F, Vélez F, VitenseK JDH, Fieberg J (2020) Computational reproducibility in the wildlife society's flagship journals. J Wildlife Manage 84:1012-1017. https://doi.org/10. 1002/jwmg. 21855

Baker M (2016) Is there a reproducibility crisis? Nature 533:452-454

Bakker TCM, Traniello JFA (2020) Ensuring data access, transparency, and preservation: mandatory data deposition for Behavioral Ecology and Sociobiology. Behav Ecol Sociobiol 74:132

Barnes N (2010) Publish your computer code: it is good enough. Nature 467:753

Buckheit JB, Donoho DL (1995) WaveLab and reproducible research. In: Antoniadis A, Oppenheim G (eds) Wavelets and Statistics. Springer-Verlag, New York, pp 55-81
Christensen G, Dafoe A, Miguel E, Moore DA, Rose AK (2019) A study of the impact of data sharing on article citations using journal policies as a natural experiment. PLoSONE 14:e0225883. https://doi.org/10.1371/journal.pone.0225883

Clark TD, Raby GD, Roche DG, Binning SA, Speers-Roesch B, Jutfelt F, Sundin J (2020a) Ocean acidification does not impair the behaviour of coral reef fishes. Nature 577:370-375

Clark TD, Raby GD, Roche DG, Binning SA, Speers-Roesch B, Jutfelt F, Sundin J (2020b) Reply to: Methods matter in repeating ocean acidification studies. Nature 586:E25-E27

Colavizza G, Hrynaszkiewicz I, Staden I, Whitaker K, McGillivray B (2020) The citation advantage of linking publications to research data. PLoS ONE 15:e0230416. https://doi.org/10.1371/ journal.pone. 0230416

Cooper N, Hsing P-Y (eds) (2017) A guide to reproducible code in Ecology and Evolution. British Ecological Society, London, https://www.britishecologicalsociety.org/wp-content/ uploads/2017/12/guide-to-reproducible-code.pdf. Accessed 12 Jan 2021

Culina A, van den Berg I, Evans S, Sánchez-Tójar A (2020) Low availability of code in ecology: a call for urgent action. PLoS Biol 18:e3000763

Farrar BG, Boeckle M, Clayton NS (2020) Replications in comparative cognition: what should we expect and how can we improve? Anim Behav Cogn 7:1-22

Fidler F, Chee YE, Wintle BC, Burgman MA, McCarthy MA, Gordon A (2017) Metaresearch for evaluating reproducibility in ecology and evolution. Bioscience 67:282-289

Fraser H, Barnett A, Parker TH, Fidler F (2020) The role of replication studies in ecology. Ecol Evol 10:5197-5207

Fraser H, Parker T, Nakegawa S, Barnett A, Fidler F (2018) Questionable research practices in ecology and evolution. PLoS ONE 13:e0200303

Grahe JE, Cuccolo K, Leighton DC, Cramblet Alvarez LD (2019) Open science promotes diverse, just, and sustainable research and educational outcomes. Psychol Learn Teach 19:5-20

Hampton SE, Anderson SS, Bagby SC et al (2015) The Tao of open science for ecology. Ecosphere 6:1-13. https://doi.org/10.1890/ ES14-00402.1

Hatchwell BJ (2017) Replication in behavioural ecology: a comment on Ihle et al. Behav Ecol 28:360. https://doi.org/10.17226/ 25303

Ihle M, Winney IS, Krysalli A, Croucher M (2017) Striving for transparent and credible research: practical guidelines for behavioral ecologists. Behav Ecol 28:348-354

Kelly CD (2019) Rate and success of study replication in ecology and evolution. PeerJ 7:e7654. https://doi.org/10.7717/peerj. 7654

Lai J, Lortie CJ, Muenchen RA, Yang J, Ma K (2019) Evaluating the popularity of $\mathrm{R}$ in ecology. Ecosphere 10:e02567. https://doi.org/ $10.1002 /$ ecs 2.2567

McKiernan EC, Bourne PE, Brown CT et al (2016) How open science helps researchers succeed. eLife 5:e16800

Mislan KAS, Heer JM, White EP (2016) Evaluating the status of code in Ecology. Trends Ecol Evol 31:4-7

Munafò MR, Nosek BA, Bishop DVM, Button KS, Chambers CD, Percie du Sert N, Simonsohn U, Wagenmakers E-J, Ware JJ, Ioannidis JPA (2017) A manifesto for reproducible science. Nat Hum Behav 1:0021. https://doi.org/10.1038/s41562-016-0021

Munday PL, Dixson DL, Welch MJ et al (2020) Methods matter in repeating ocean acidification studies. Nature 586:E20-E24

National Academies of Sciences, Engineering, and Medicine (2019) Reproducibility and replicability in science. The National Academies Press, Washington DC 
Noble WS (2009) A quick guide to organizing computational biology projects. PLoS Comput Biol 5:e1000424. https://doi.org/10.1371/ journal.pcbi.1000424

Nosek BA, Spies JR, Motyl M (2012) Scientific utopia: II. Restructuring incentives and practices to promote truth over publishability. Perspect Psychol Sci 7:615-631

O'Grady C (2020) Ecologists push for more reliable research. Science 370:1260-1261

Open Science Collaboration (2015) Estimating the reproducibility of psychological science. Science 394:aac4716. https://doi.org/10. $1126 /$ science.aac 4716

Parker TH (2013) What do we really know about the signaling role of plumage colour in blue tits? A case study of impediments to progress in evolutionary biology. Biol Rev 88:511-536

Perkel JM (2019) Paper lets scientists play with each other's results. Nature 567:17-18

Piwowar HA, Day RS, Fridsma DB (2007) Sharing detailed research data is associated with increased citation rate. PLoS ONE 2:e308. https://doi.org/10.1371/journal.pone.0000308

Piwowar HA, Vision TJ (2013) Data reuse and the open data citation advantage. PeerJ 1:e175

Powers SM, Hampton SE (2019) Open science, reproducibility, and transparency in ecology.Ecol Appl 29:e01822. https://doi.org/10. 1002/eap.1822

Roche DG, Kruuk LEB, Lanfear R, Binning SA (2015) Public data archiving in Ecology and Evolution: how well are we doing?
PLoSBiol 13:e1002295. https://doi.org/10.1371/journal.pbio. 1002295

Sandve GK, Nekrutenko A, Taylor J, Hovig E (2013) Ten simple rules for reproducible computational research. PLoS Comput Biol 9:e1003285

Stevens JR (2017) Replicability and reproducibility in comparative psychology. Front Psychol 8:862

Van Lissa CJ, Brandmaier AM, Brinkman L, Lamprecht A, Peikert A, Struiksma ME, Vreede B (2020) WORCS: a workflow for open reproducible code in science. PsyArXiv, https://doi.org/10.31234/ osf.io/k4wde

Wessel L (2020) After scalding critiques of study on gender and mentorship, journal says it is reviewing the work. Science, https://doi. org/10.1126/science.abf8164

Wilkinson MD, Dumontier M, Aalbersberg IJ et al (2016) The FAIR guiding principles for scientific data management and stewardship. Sci Data 3:160018. https://doi.org/10.1038/sdata.2016.18

Publisher's note Springer Nature remains neutral with regard to jurisdictional claims in published maps and institutional affiliations. 